\title{
E-Santé et innovation thérapeutique
}

Sabrina Sacconi, Judith Mehl, Claire Lenain, Frédéric Lofaso, Jessica Taytard

\begin{abstract}
> L'utilisation en santé des outils numériques ouvre un large champ d'innovation non médicamenteuse synonyme d'avancées pour les patients, leurs familles et les professionnels de santé, notamment en matière de suivi respiratoire, crucial pour les maladies neuromusculaires. Reste à surmonter des problématiques spécifiques, notamment éthiques. <
\end{abstract}

\section{Des initiatives multiples au CHU de Nice}

\section{Sabrina Sacconi}

Coordinatrice adulte du Centre de référence des maladies

neuromusculaires, Nice, France.

SACCONI.S@chu-nice.fr

L'essor de l'usage des nouvelles technologies en santé représente un espoir. II suscite aussi un certain nombre de questions, à commencer par ses bénéfices effectifs pour le patient, avec la crainte pour certains d'une perte de contact, voire d'une déshumanisation. La première Journée d'information sur la e-santé au service des maladies chroniques neuromusculaires et neurogénétiques ${ }^{1}$ organisée le 19 janvier 2018 a rassemblé au CHU de Nice plus de 200 participants. La thématique suscite donc l'intérêt des professionnels de santé et des patients, comme elle suscite celui d'acteurs publics et privés à l'exemple des laboratoires pharmaceutiques. Les maladies neuromusculaires représentent en effet un modèle intéressant de développement de la e-santé, qui pourrait être applicable à la population vieillissante. Au CHU de Nice [1], la télémédecine fait déjà l'objet de plusieurs appels d'offres en cours, pour favoriser la prise en charge des patients à domicile ou dans les hôpitaux de proximité et ainsi réduire leurs déplacements. Dans le cadre du projet Living NMD hospital, nous avons également utilisé les nouvelles technologies pour équiper une chambre d'hôpital de semaine. Les patients y disposent d'une tablette numérique qui leur permet de contrôler l'environnement (store, position du lit...) et d'appeler les infirmiers. Elle leur permettra bientôt de se déplacer sans aide, par exemple pour aller aux toilettes. Un tel dispositif améliore le confort et l'autonomie des patients hospitalisés en même temps qu'il libère du temps au personnel soignant. Nous développons aussi un outil qui

${ }^{1}$ Colloque organisé par le Centre de référence des maladies neuromusculaires de Nice, l'European Reference Networks, la filière Filnemus, la filière Filslan, le CHU de Nice et l'AFM-Téléthon.

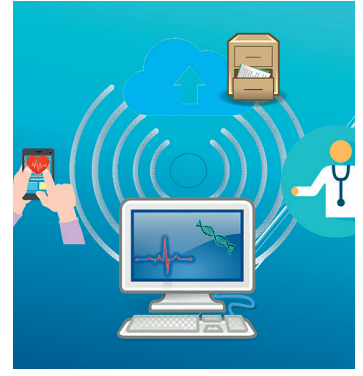

médecine/sciences $2019 ; 35$ (hors série $n^{\circ} 1$ ) : $42-5$ utilise des mesures non invasives pour le diagnostic des maladies neuromusculaires et pourra être utilisé à distance. Le CHU de Nice participe enfin au projet européen de télémédecine NMD-care, pour la prise en charge des patients transfrontaliers (Italie, France) ou habitant une zone isolée (en Corse notamment) et atteints d'une maladie neuromusculaire. $\diamond$

Multiple initiatives at the University Hospital of Nice

\section{Une régulation positive pour limiter les risques}

Judith Mehl

Vice-présidente d'Ethik-IA, France.

mehljudith@gmail.com

L'expression « intelligence artificielle » (IA) a fait son apparition en 1956. II s'agit de la science dont le but est de faire effectuer à une machine des tâches que l'homme accomplit en utilisant son intelligence. Elle a beaucoup évolué ces dernières années, pour passer à I'IA dite « apprenante », qui apprend de son environnement et de ses erreurs pour améliorer ses performances à l'exemple de l'ordinateur qui a battu un champion du jeu de $\mathrm{Go}^{2}$. Des systèmes d'IA existent déjà en santé, notamment dans le diabète de type 1 où des algorithmes calculent, à partir des données fournies par un capteur de glycémie, la dose d'insuline à injecter au patient à chaque instant. Des solutions existent dans d'autres pays, notamment en

${ }^{2}$ En 2015, le programme AlphaGo de Google DeepMind a battu pour la première fois au jeu de go le champion Fan Hui. 


\section{Les 5 cinq clés de régulation positive d'Ethik-IA}

1. Consentement et information du patient.

2. Garantie humaine de l'intelligence artificielle.

3. Gradation de la régulation en fonction du niveau de sensibilité des données.

4. Accompagnement de l'adaptation des métiers.

5. Intervention d'une supervision externe indépendante.

ophtalmologie. Aux États-Unis, la Food and Drug Administration (FDA) a récemment donné son approbation pour l'utilisation d'une IA dans le diagnostic de la rétinopathie diabétique. Ces évolutions ont été rendues possibles par l'accélération de la puissance de calcul des machines et l'augmentation de la masse de données (big data). La collecte de ces données et leur utilisation s'inscrivent dans un cadre juridique existant, avec notamment les lois de bioéthique françaises et le Règlement général sur la protection des données (RGPD) européen. A l'évidence, le big data constitue un vecteur majeur d'avancées en termes de santé publique, tant en matière d'épidémiologie que de qualité de soins, de parcours de soins et de connaissances scientifiques. Dans le même temps, I'IA mise au service de la santé fait peur. Son déploiement présente des enjeux éthiques et humains majeurs qu'il faut anticiper. C'est l'objet de la réflexion menée par Ethik-IA, ainsi il faut impérativement réguler de manière positive le déploiement de l'IA et de la robotisation en santé, à la fois pour éviter un millefeuille administratif défavorable au développement de solutions en France et pour disposer de solutions éthiquement acceptables.

La troisième de ces clés concerne la nécessité d'un traitement différencié selon la sensibilité des données. Sur ce sujet, nous travaillons actuellement avec l'Institut-Hospitalo-Universitaire (IHU) Imagine pour émettre des normes sur l'application de l'IA aux données génétiques, évaluer la sensibilité des données et de leur exploitation possible. Au même titre que les agents infectieux sont manipulés avec des niveaux de sécurité biologiques différents (P1 à P4) selon leur dangerosité, les données de santé pourraient bénéficier d'un système similaire, fonction de leur sensibilité, les plus sensibles aujourd'hui étant les données génétiques. $\diamond$ A positive regulation to limit risks

\section{Des enjeux de sécurité et d'interopérabilité}

\section{Claire Lenain}

Directrice de l'offre de service et de la communication ASIP Santé,

France.

Claire.LENAIN@sante.gouv.fr

L'usage des outils numériques dans le champ de la santé et du médicosocial permet d'améliorer le quotidien des professionnels de santé et des patients (gain de temps, aide diagnostique...), de favoriser la qualité et la sécurité des soins, d'améliorer l'efficience du système de santé (par exemple en évitant les actes redondants) et de favoriser l'égalité d'accès aux soins en tous points du territoire. La e-santé est donc une opportunité, mais elle doit se développer dans un cadre maitrisé. Mettre en œuvre ce cadre de la e-santé est l'une des missions principales, avec la réalisation de projets de transformation numérique, de l'Agence française de la santé numérique (ASIP Santé). Cet opérateur public agit pour le compte du ministère en charge de la santé, de l'Assurance maladie et de la Caisse nationale de solidarité pour l'autonomie (CNSA). Les données de santé sont sensibles et personnelles (antécédents, traitements en cours ). II ne faut ni les perdre, ni les dénaturer. Elles doivent être stockées dans un espace sécurisé, d'abord dans les logiciels utilisés en permanence par les professionnels de santé (le logiciel du cabinet du professionnel libéral, le système d'information de l'établissement ) puis dans les outils qui facilitent le partage de documents médicaux comme le dossier médical partagé (DMP), et rester accessibles aux seules personnes habilitées. Le DMP, dont la Caisse nationale d'Assurance maladie a annoncé récemment le déploiement généralisé sur tout le territoire, a vocation à être un entrepôt sécurisé national contenant des documents de santé utiles à la coordination des soins. L'interopérabilité entre les systèmes d'information de santé permet aux données de circuler de façon fluide et sécurisée, afin d'être partagées entre les intervenants du parcours de soins. L'ASIP Santé met à disposition, sur son site Internet ${ }^{3}$, un cadre d'interopérabilité des systèmes d'information de santé (CI-SIS) (Figure 1). II contient des spécifications standards que peuvent utiliser les industriels pour proposer aux professionnels de santé des interfaces interopérables, et donc communicantes. Ce cadre a été créé en 2009, initialement pour favoriser le partage d'informations entre l'hôpital et la ville dans la perspective du déploiement du DMP. II s'est ensuite étendu à d'autres champs. L'ASIP Santé est également très active en matière de référentiels de sécurité. Nous travaillons avec les industriels qui développent des solutions de e-santé pour s'assurer du respect des bonnes pratiques et de la réglementation, et donc du cadre de la e-santé. Par ailleurs, les pouvoirs publics mettent en place au niveau national des services pour faciliter l'échange et le partage d'informations qui nous concernent, en tant que patients. Par exemple, les messageries sécurisées de santé permettent l'échange de données de santé entre professionnels de santé. Des services complémentaires sont portés par les Agences régionales de santé (ARS), avec l'aide de groupements régionaux d'appui au développement de la e-santé. $\diamond$

Security and interoperability issues

${ }^{3}$ http://esante.gouv.fr 


\section{La télésurveillance respiratoire, un outil en devenir}

\section{Frédéric Lofaso}

Chef du service Physiologie et exploration fonctionnelle,

Hôpital Raymond Poincaré, Garches, France.

f.lofaso@aphp.fr

L'un des principaux risques vitaux dans les maladies neuromusculaires est la décompensation respiratoire aiguë. Elle peut être fatale ou entrainer une hospitalisation en réanimation avec intubation. Les patients que nous voyons en consultation sont désormais plus souvent sous ventilation non invasive (VNI) que trachéotomisés. Certains sont totalement dépendants de leur ventilateur. Ils utilisent le jour une pipette buccale, et la nuit un masque nasal. Ce sont les plus à risque d'encombrement et de décompensation. Ce risque reste difficile à évaluer lors d'une consultation à l'hôpital, tous les six mois ou tous les ans. La première étude à avoir démontré l'intérêt du suivi à distance de patients insuffisants respiratoires est parue en 2009 [2]. Elle a montré qu'un suivi à domicile quotidien de la saturation en oxygène entrainait une réduction du nombre de jours d'exacerbation. De nombreuses études, aux résultats contradictoires, sont parues depuis. En 2018, une revue de la littérature a conclu à l'intérêt de la télémédecine pour éviter une décompensation respiratoire sur des populations bien ciblées (pathologie, âge, sévérité) [3]. Reste que nous avons peu de possibilités d'opérer ce type de suivi pour nos patients ventilés. Les ventilateurs enregistrent, certes, des données: fuites, apnées obstructives, fréquence respiratoire, saturation en oxygène pour certains, mouvements thoraco-abdominaux (via des sangles avec capteurs) pour d'autres... Ces informations sont récupérées par les prestataires de ventilation à domicile. Ils nous les envoient de façon régulière mais nous n'avons pas le temps et ne sommes pas rétribués pour les analyser au quotidien. Nous ne pouvons que nous contenter de les utiliser pour des travaux de recherche. Dans l'une de nos études, l'enregistrement à domicile de données simples (débit, pression, synchronisation ventilateur/ patient, saturation en oxygène...) de patients sous ventilation buccale a permis d'optimiser leur ventilation [4]. Un tel suivi est actuellement impossible en routine. Il serait pourtant utile pour évaluer les patients ventilés en conditions de vie réelle, entre deux consultations. Il pourrait également éviter aux patients, qui sont lourdement handicapés, d'être hospitalisés 48 heures pour une simple polysomnographie, nécessaire pour s'assurer qu'ils sont bien ventilés la nuit. Enfin, le suivi à domicile pourrait être utilisé pour ajuster la ventilation à distance, une possibilité désormais offerte par certains ventilateurs. Nous menons actuellement une étude sur ce thème. $\nabla$ Innovation in telemonitoring respiratory disturbances

\section{Une application mobile à la main des adolescents}

\section{Jessica Taytard}

Service de Pneumologie pédiatrique de l'Hôpital Armand

Trousseau, Paris, France.

jessica.taytard@trs.aphp.fr

En pédiatrie, il existe des difficultés de communication entre les différents acteurs de la prise en charge et un risque de perte d'informations sur le quotidien et la qualité de vie des patients ventilés, en particulier quand il s'agit d'adolescents. Cette problématique nous a conduits à développer I'application OVNI, pour Optimisation de la Ventilation Non Invasive. Le choix de cet outil tient à son caractère évolutif, personnalisable et ludique, qui favorise son utilisation par les adolescents et s'appuie sur un support (le Smartphone) qu'ils ont toujours sur eux, y compris en consultation. Ce projet a été primé au RespirH@aktion 2016,un hackathon d'un week-end sur les maladies respiratoires au cours duquel nous avons pu rencontrer des développeurs, des graphistes et des sponsors. Sur l'application ONVI, l'adolescent crée son profil et peut personnaliser son interface.

\section{Trois problématiques évoquées lors de l'atelier}

- Les patients doivent être largement impliqués dans l'essor de la e-santé. Tout projet d'outil numérique qui leur est destiné doit partir des besoins exprimés par les patients et les intégrer à chaque étape du développement.

- Les autorités de santé régulent l'accès au marché des médicaments. Une régulation comparable s'avère plus compliquée pour l'intelligence artificielle. Elle pourrait passer par des tests fonctionnels des algorithmes.

- Une interopérabilité effective des systèmes d'information permettrait aux professionnels de santé de gagner un temps précieux, par exemple dans un contexte d'urgence, ou pour remplir tout au long du suivi une base de données à partir du dossier patient. Elle se heurte actuellement à un manque de moyens, notamment pour mettre à jour le matériel informatique, parfois vétuste, des établissements de santé. Elle se heurte également à un verrouillage des systèmes d'information hospitaliers, et des machines de ventilation, par crainte d'actions malveillantes sur des données sensibles. Pour contourner cet écueil, certains établissements se rendent interopérables via des systèmes externes. 

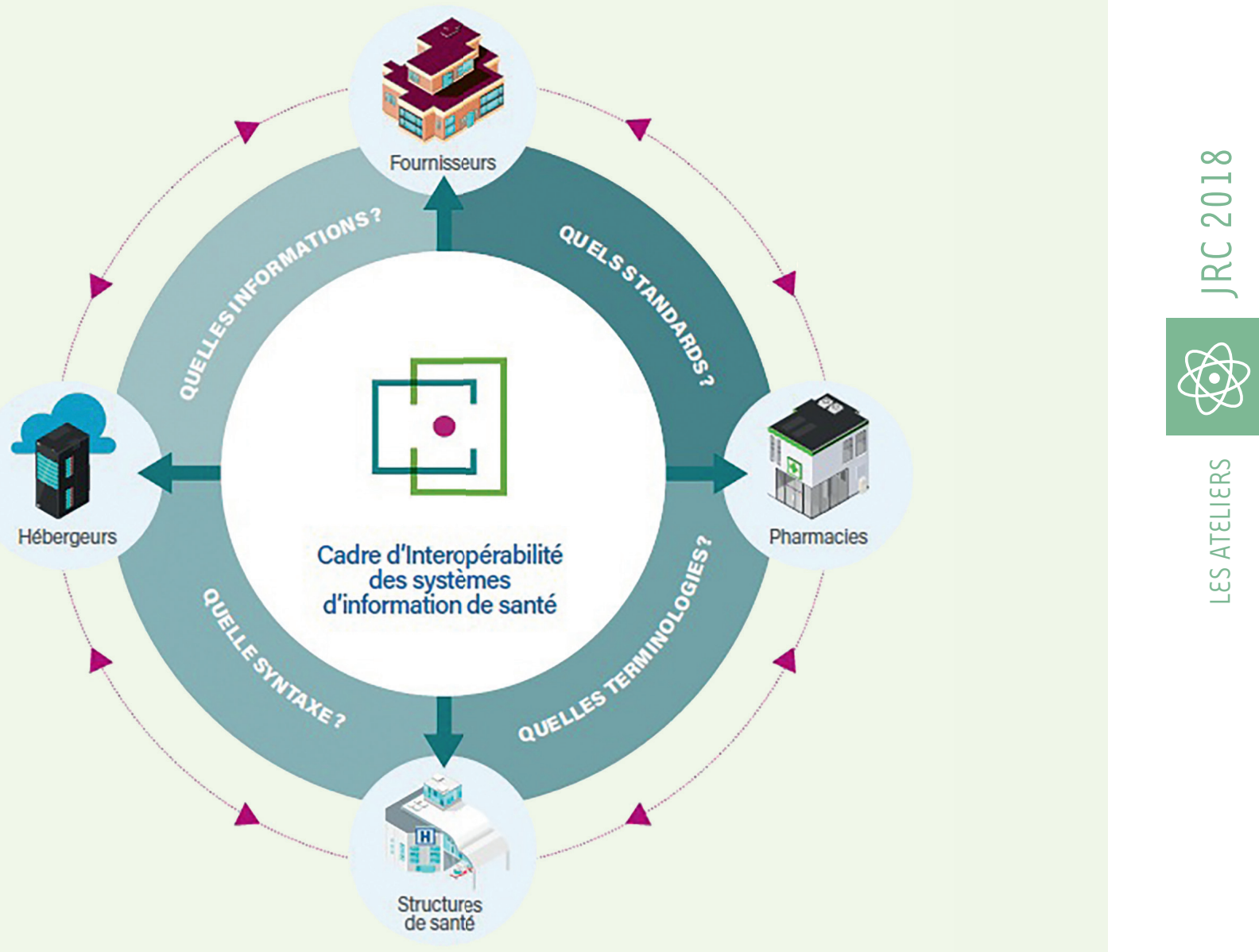

Figure 1. Le Cadre d'Interopérabilité des systèmes d'information de santé. Le Cadre d'Interopérabilité est un ensemble de spécifications qui définit les informations à échanger entre les systèmes d'information de santé et identifie le standard le plus approprié à utiliser pour cet échange (source: ASIP Santé).

Il répond à des notifications sur l'utilisation de sa ventilation non invasive (nombre d'heures) et la qualité de son sommeil. II peut ajouter des notes, inscrire ses contacts (prestataires, médecin d'urgence), gérer ses rendez-vous, accéder à ses statistiques et les montrer (ou non) à son médecin en consultation. Nous commençons à proposer notre application aux patients et attendons leurs retours. $\diamond$

A mobile application to help adolescent patients

\section{LIENS D'INTÉRÊT}

Les auteurs déclarent n'avoir aucun lien d'intérêt concernant les données publiées dans cet article.

\section{RÉFÉRENCES}

1. Puma A, Villa L, Soriani MH, et al. E-health and innovation to overcome barriers in neuromuscular diseases. European reference network EURO-NMD annual meeting 2017, poster (sur https://ern-euro-nmd.eu/).

2. Vitacca M, Bianchi L, Guerra A, et al. Tele-assistance in chronic respiratory failure patients: a randomised clinical trial. Eur Respir/ 2009 ; 33 : 411-8.

3. Vitacca M, Montini A,' Comini L. How will telemedicine change clinicial practice in chronic obtructive pulmonary disease. Ther Adv Respir Dis 2018 ; 12 : 1753465818754778 .

4. Nardi J, Leroux K, Orlikowski D, et al. Home monitoring of daytime mouthpiece ventilation effectiveness in patients with neuromuscular disease. Chron Respir Dis 2016 ; $13: 67-74$.

$\varepsilon$-Health and therapeutic innovation

Retrouvez toutes les Actualités de la Myologie sur les sites de :

la Société Française de Myologie www.sfmyologie.org

la filière de santé neuromusculaire FILNEMUS

www.filnemus.fr
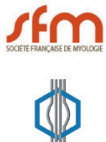

Filnemus 